\title{
Low-intensity shock wave therapy for erectile dysfunction and the influence of disease duration
}

\author{
Pedro Simoes de Oliveira, Tiago Ribeiro de Oliveira, Álvaro Nunes, Francisco Martins, Tomé Lopes \\ SCI-Centro de Urologia, Lisbon, Portugal.
}

\begin{abstract}
Summary Objective: Low-intensity shock-wave treatment (LiSWT) is a therapy for erectile dysfunction (ED) with good results reported in the literature. The aim of this study was to evaluate the results of LiSWT on patients treated for ED and the influence of ED duration in treatment outcomes.

Material and methods: We performed an open-label single-arm prospective study of patients treated with LiSWT for ED.

Patients were assessed with the IIEF-5 at baseline and at six weeks and three months after LiSWT, and with penile dynamic Doppler ultrasound before treatment and six weeks after. Patients were divided into two groups accordingly to ED evolution time: $\leq 24$ months and $>24$ months.

Results: Twenty-five patients were enrolled, 13 had ED $\leq 24$ months and $12>24$ months. Median baseline IIEF-5 was 14, at 6 weeks post LiSWT was $16(p<0.001)$ and at 3 months post LiSWT was $18(p<0.001)$. Mean baseline peak systolic velocity (PSV) was $29.3 \pm 13.0 \mathrm{~cm} / \mathrm{s}$, after LiSWT was $35.9 \pm 15.2$ $\mathrm{cm} / \mathrm{s}$ ( $p$ 0.001). Mean baseline end-diastolic velocity (EDV) was $2.6 \pm 4.8 \mathrm{~cm} / \mathrm{s}$ and after LiSWT was $1.3 \pm 4.3 \mathrm{~cm} / \mathrm{s}(p 0.015)$. No statistical significative difference was identified between the two groups.

Conclusions: LiSWT is a safe, harmless and repeatable treatment tool for ED with good outcomes reported. Our results suggest that length of disease duration doesn't negatively influences treatment results.
\end{abstract}

KEY WORDS: Erectile dysfunction; Penis; Shock wave therapy; Time-to-treatment; Treatment outcome.

Submitted 4 October 2018; Accepted 15 November 2018

\section{INTRODUCTION}

Erectile dysfunction (ED) is a common condition affecting more than $50 \%$ of men aged $40-70$ years (1). Available treatments include phosphodiesterase type 5 inhibitors (PDE5i), vacuum devices, topical, intraurethral or intracavernosal, administration of vasoactive agents or, in the most severe cases, penile prosthesis. Although many patients are satisfied with these treatments, others are not, due to poor response or impossibility of using them. Low-intensity shock wave therapy (LiSWT) is another available first line therapy for ED. Since Vardi et al. (2) first described its use on ED, several reports have been published with encouraging results. Although the mechanism of action is poorly understood, it is suggested that LiSWT can induce neovascularization, anti-inflammation and tissue regeneration leading to structural changes and, therefore improvement in erectile function (3). Published studies have different samples, different protocols and different inclusion criteria. There is still no evidence of which patient is the best candidate for LiSWT. The aim of our study was to evaluate the results of LiSWT on patients treated for ED and looking for cofounding factors that could influence treatment outcomes, specially duration of ED.

\section{Materials AND MEthods}

We performed an open-label single-arm prospective study of all patients who underwent LiSWT for ED, at a single center from June 2016 to March 2018. Patients were assessed with the simplified International Index of Erectile Function (IIEF-5) before starting the treatment and at six weeks and three months after. Assessment included also penile dynamic Doppler ultrasound (PDDU) before treatment and six weeks after. Inclusion criteria included, age over 18 years-old, a total IIFE-5 score $<22$, no psychiatric disturbance and no active skin lesion at the treatment site. Treatment was performed using the PiezoWave2 (Richard Wolf GmbH, Knittlingen, Germany) device with a linear probe. Treatment protocol included a weekly session for six weeks. Each session delivered 2000 shocks on the perineum plus 2000 shocks on dorsum penis with an energy flux density (EFD) of $0.160 \mathrm{~mJ} / \mathrm{mm}^{2}$. During treatment every patient had tadalafil $5 \mathrm{mg}$ daily. Patients were divided into two groups accordingly to ED evolution time, defined by time-to-treatment since the beginning of symptoms: $\leq 24$ months (group 1) and > 24 months (group 2).

Other analyzed variables included, age, type of ED (arteriogenic, arteriogenic + venous leak, post radical prostatectomy and, venous leak), ED risk factors and PDE5i treatment necessity and response.

An increase in the IIEF-5 after LiSWT was considered "improvement". Regarding PDDU, an increase in peak systolic velocity (PSV) and/or decrease in end-diastolic velocity (EDV) after LiSWT was considered "improvement". Regarding PDE5i treatment, "improvement" was defined when a patient previously on PDE5i, was able to leave medication. "Improvement" in PDEi5 response was considered whenever a patient subjectively improved the response to medication after LiSWT considering three categories: "good", "moderate" and "bad". 
The primary endpoint was any observed change in IIEF-5 and PDDU associated with LiSWT and comparing results between groups 1 and 2 regarding the influence of disease duration in treatment response.

The secondary endpoint was to evaluate the response to treatment with PDE5i associated with LiSWT and comparing results between groups 1 and 2. Also, LiSWT results were evaluated accordingly with ED type and risk factors. Adverse events, patient satisfaction and recommendation were also assessed.

Clinical data was analyzed using IBM SPSS Statistics, version 24.0 (IBM Corp., Armonk, NY, USA). Descriptive statistics were reported as frequencies for categorical variables and, mean, median (first quartile - third quartile) and standard deviation for continuous variables. Comparison between pre-treatment and posttreatment and between groups 1 and 2 results was performed using the Wilcoxon Signed-Rank test. X2-test (two-sided Pearson X2-test with two degrees of freedom) was used between IIEF-5, PDDU, PDE5i, ED type and ED risk factors. Fisher's exact test was used when the expected frequency was of five or less. Statistical significance was considered for $p$ values $<0.05$.

\section{RESULTS}

Twenty-five patients were enrolled, 13 had ED $\leq 24$ months (group 1) and $12>24$ months (group 2). Fifteen patients had arteriogenic $\mathrm{ED}$, four arteriogenic and venous leak ED, three post-radical prostatectomy ED and, three venous leak. Median age was 61 years-old (range: 27-73).

Patient demographics are described in Table 1.

Table 2 shows the results of the total study sample. Median baseline IIEF- 5 was 14, at 6 weeks post LiSWT was $16(p<0.001)$ and at 3 months post LiSWT was 18 ( $\mathrm{p}<$ 0.001 ), with an improvement of $68 \%$ and $72 \%$ respectively. Mean baseline PSV was $29.3 \pm 13.0 \mathrm{~cm} / \mathrm{s}$, after LiSWT was $35.9 \pm 15.2 \mathrm{~cm} / \mathrm{s}$ (p 0.001) representing an 84\% improvement. Mean baseline EDV was $2.6 \pm 4.8 \mathrm{~cm} / \mathrm{s}$, after LiSWT was $1.3 \pm 4.3 \mathrm{~cm} / \mathrm{s}$ (p 0.015) representing an $68 \%$ improvement. There was no significative result in PDE5i treatment, nevertheless, PDE5i response had an improvement of 36\% (p 0.004).

Tables $2 \mathrm{a}$ and $2 \mathrm{~b}$ show the specific results of group 1 and group 2 respectively and separately.

Table 2.

Results.
Table 1.

Patient demographics.

\begin{tabular}{|c|c|c|c|}
\hline \\
\hline \multicolumn{4}{|l|}{$\frac{\text { lotal number }}{25 \text { patients total }}$} \\
\hline \multicolumn{4}{|l|}{13 patients $\mathrm{ED} \leq 24$ months } \\
\hline \multicolumn{4}{|l|}{12 patients ED > 24 months } \\
\hline Age at LiSWT (years) & Median (range) & & \\
\hline Total & $61(27-73)$ & & \\
\hline $\mathrm{ED} \leq 24$ months & $56(42-73)$ & & \\
\hline ED > 24 months & $62.5(27-73)$ & & \\
\hline ED type & $\begin{array}{l}\mathrm{n}(\%) \\
\text { Total }\end{array}$ & $\begin{array}{c}n(\%) \\
E D \leq 24 \text { months }\end{array}$ & $\begin{array}{c}n(\%) \\
\text { ED > } 24 \text { months }\end{array}$ \\
\hline Arteriogenic & $15(60)$ & $9(69)$ & $6(50)$ \\
\hline Arteriogenic + venous leak & $4(16)$ & $1(8)$ & $3(25)$ \\
\hline Post-radical prostatectomy & $3(12)$ & $2(15)$ & $1(8)$ \\
\hline Venous leak & $3(12)$ & $1(8)$ & $2(17)$ \\
\hline ED risk factors & $\begin{array}{l}\text { n (\%) } \\
\text { Total }\end{array}$ & $\begin{array}{c}n(\%) \\
\text { ED } \leq 24 \text { months }\end{array}$ & $\begin{array}{c}n(\%) \\
\text { ED > } 24 \text { months }\end{array}$ \\
\hline Hypertension & $16(64)$ & $8(62)$ & $8(67)$ \\
\hline Dyslipidemia & $15(60)$ & $8(62)$ & $7(58)$ \\
\hline Diabetes & $7(28)$ & $5(39)$ & $2(17)$ \\
\hline Tobacco & $5(20)$ & $4(31)$ & $1(8)$ \\
\hline Obesity & $8(32)$ & $5(39)$ & $3(25)$ \\
\hline ED evolution time & Median (months) & Range (months) & \\
\hline Total & 24 & $5-192$ & \\
\hline $\mathrm{ED} \leq 24$ months & 18 & $5-24$ & \\
\hline ED $>24$ months & 66 & $30-192$ & \\
\hline
\end{tabular}

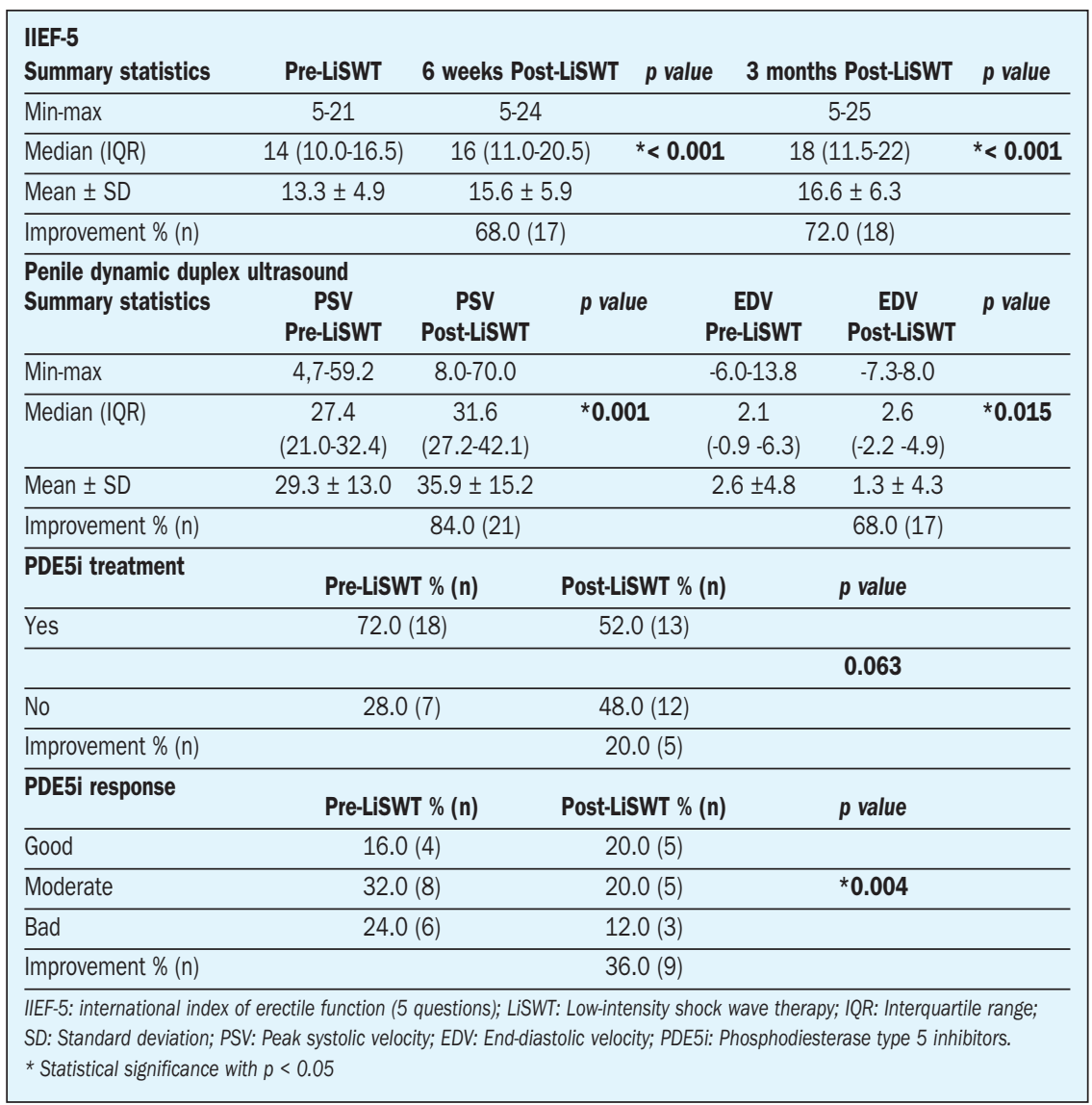


Table 2a.

Results $E D \leq 24$ months.

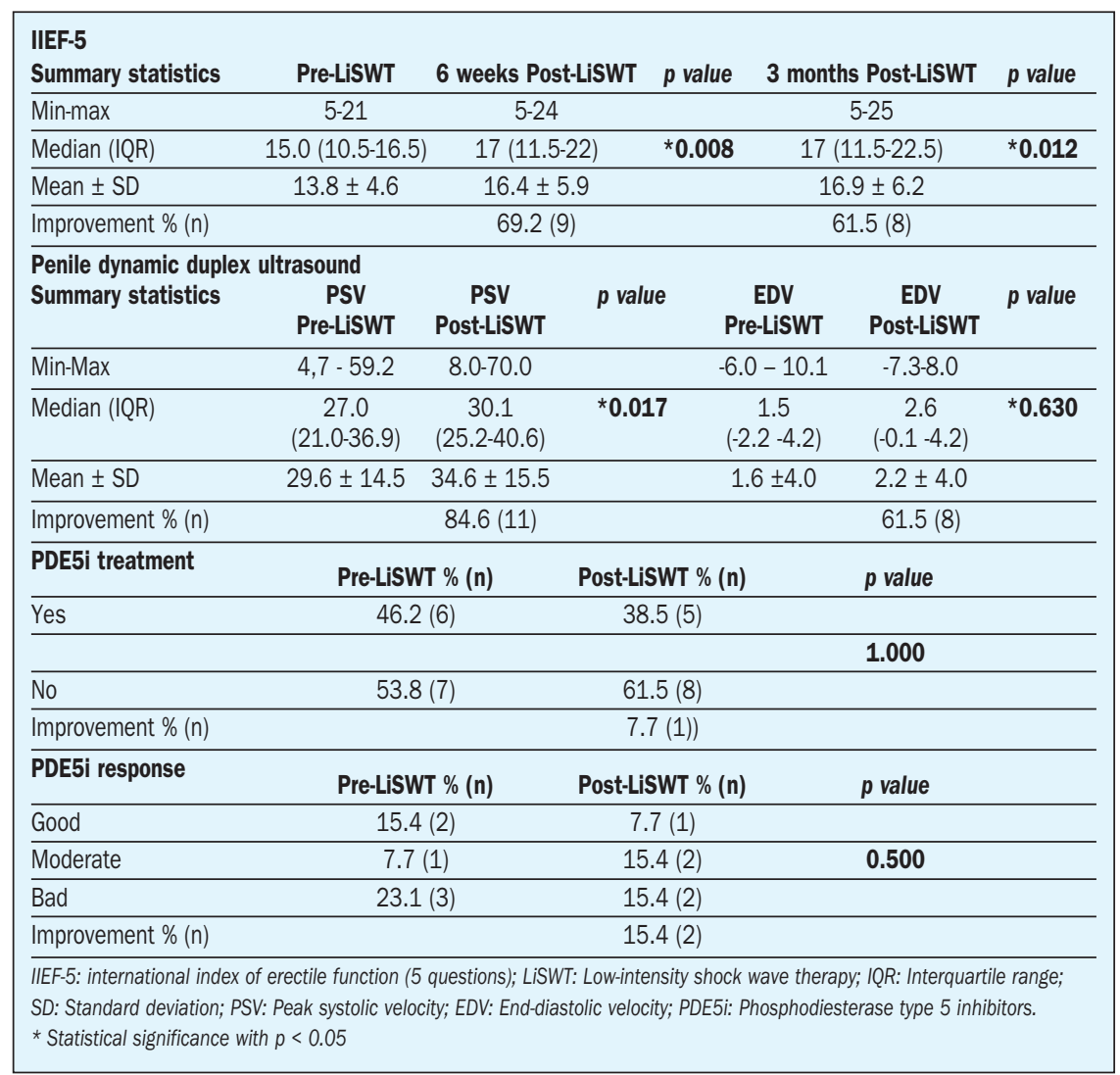

\section{Table $2 b$.}

Results ED > 24 months.

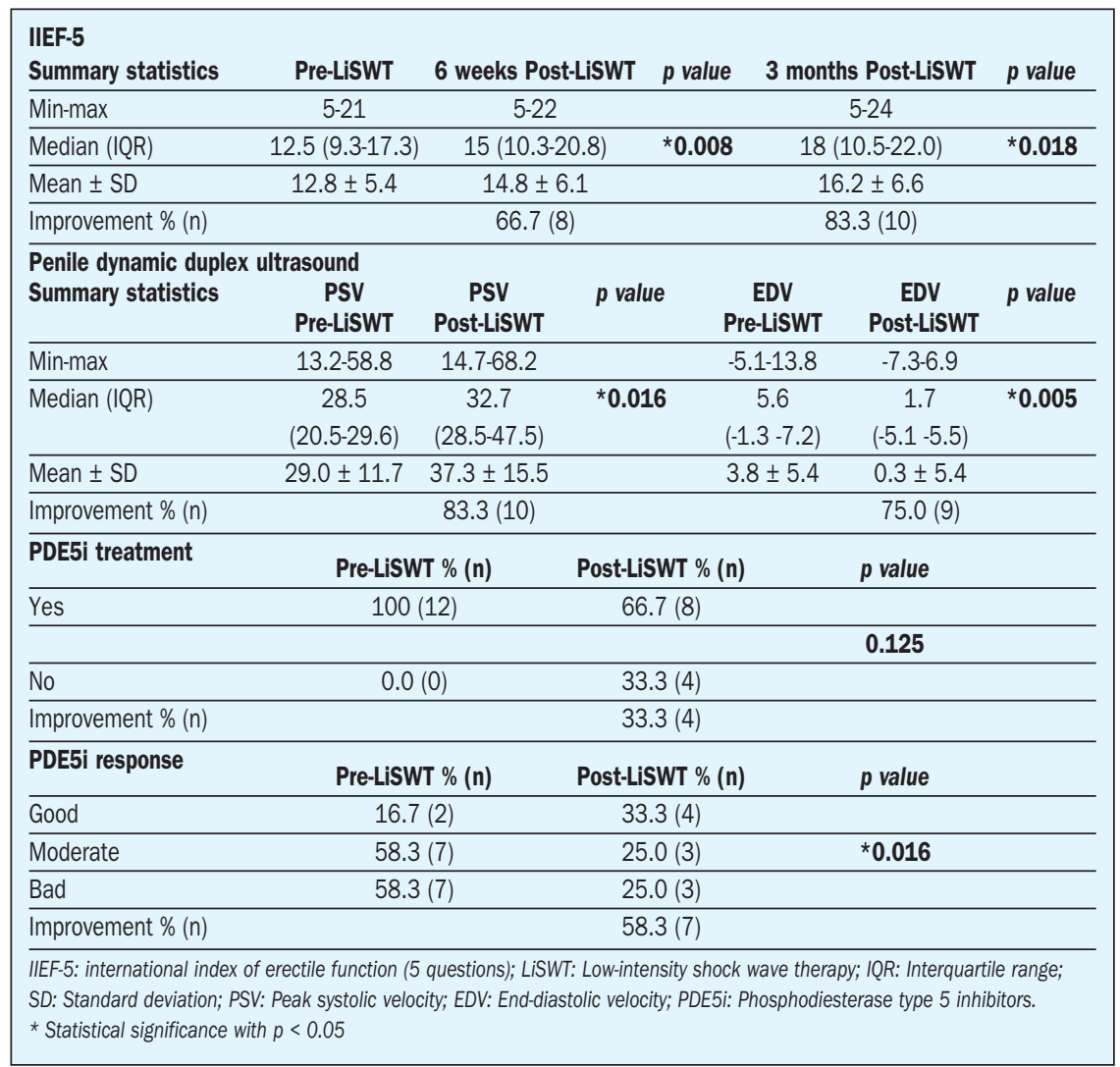

Table 3 shows the results of the total study sample accordingly with type of ED. Statistical significance was seen only in the IIEF-5 at 6 weeks after LiSWT, presenting the best response patients with arteriogenic and/or venous leak ED (p 0.021).

Table 4 shows the results of the total study sample accordingly with ED risk factors. Statistical significance was seen in the PDE5i response improvement, where diabetic patients presented the worse response ( $p$ 0.027)

Table 5 compares the results and treatment improvement between the two groups. No statistical significative difference was identified beside a better PDE5i response in patients with ED > 24 months. At the end of the study, overall patient satisfaction with LiSWT was $76 \%$ and, $80 \%$ of patients would recommend it (Table 6). No adverse effect was reported.

\section{Discussion}

ED is a common medical condition and epidemiological data have shown a high incidence and prevalence worldwide (1). This greatly disseminated and progressive condition has great impact in patient's quality of life and it's no wonder efforts have been made in order to find a successful treatment.

Although the true mechanism of action is not well understood, according to basic science evidences it can be hypothesized that LiSWT may act by several pathways leading to cell proliferation, angiogenesis, nerve regeneration and anti-inflammation (3). It is theorized that energy carried by LiSWT compresses the tissue and the following negative pressure originates tensile forces leading to shear stress on cell membranes. This phenomenon is called "cavitation" and triggers a chain of events that cause the release of angiogenic factors such as endothelial NO synthase, vascular endothelial growth factor and proliferating cell nuclear antigen (3)

Following this rationale, Vardi et al pioneered the first study using LiSWT for ED. Twenty men with vasculogenic ED were included in their study: at one-month post- 
Table 3.

Results by ED type.

\begin{tabular}{|c|c|c|c|c|}
\hline IIEF-5 improvement & 6 weeks Post-LiSWT & $p$ value & 3 months Post-LiSWT & $p$ value \\
\hline Arteriogenic & $80.0(12)$ & & $73.3(11)$ & \\
\hline Arteriogenic + venous leak & $50.0(2)$ & $\star 0.021$ & $75.0(3)$ & 0.459 \\
\hline Post-RP & $0.0(0)$ & & $33.3(1)$ & \\
\hline Venous leak & $100(3)$ & & $100(3)$ & \\
\hline \multicolumn{5}{|c|}{ Penile dynamic duplex ultrasound improvement } \\
\hline & PSV Post-LiSWT \% (n) & $p$ value & EDV Post-LiSWT \% (n) & $p$ value \\
\hline Arteriogenic & $86.7(13)$ & & $60.0(9)$ & \\
\hline Arteriogenic + venous leak & $75.0(3)$ & 0.532 & $100(4)$ & 0.133 \\
\hline Post-RP & $66.7(2)$ & & $33.3(1)$ & \\
\hline Venous leak & $100(3)$ & & $100(3)$ & \\
\hline \multicolumn{5}{|c|}{ PDE5i treatment improvement } \\
\hline Arteriogenic & 20.0 & 3 & & \\
\hline Arteriogenic + venous leak & 25.0 & 1 & 1.000 & \\
\hline Post-RP & 0 & 0 & & \\
\hline Venous leak & 33.3 & 1 & & \\
\hline \multicolumn{5}{|l|}{ PDE5i response improvement } \\
\hline Arteriogenic & 20.0 & 3 & & \\
\hline Arteriogenic + venous leak & 75.0 & 3 & 0.119 & \\
\hline Post-RP & 33.3 & 1 & & \\
\hline Venous leak & 66.7 & 2 & & \\
\hline \multicolumn{5}{|c|}{$\begin{array}{l}\text { ED: Erectile dysfunction; IIEF-5: international index of erectile function (5 questions); LiSWT: Low-intensity shock wave therapy; } \\
\text { Post-RP: Post radical prostatectomy; PSV: Peak systolic velocity; EDV: End-diastolic velocity; PDE5i: Phosphodiesterase type } 5 \\
\text { inhibitors. } \\
\text { * Statistical significance with } p<0.05\end{array}$} \\
\hline
\end{tabular}

Table 4.

Results by ED risk factor.

\begin{tabular}{|c|c|c|c|c|}
\hline IIEF-5 improvement & 6 weeks Post-LiSWT & $p$ value & 3 months Post-LiSWT & $p$ value \\
\hline HTA & $58.8(10)$ & 0.661 & $55.6(10)$ & 0.208 \\
\hline Diabetes & $29.4(5)$ & 1.000 & $22.2(4)$ & 0.355 \\
\hline Tobacco & $17.6(3)$ & 1.000 & $16.7(3)$ & 0.597 \\
\hline Dyslipidemia & $58.8(10)$ & 1.000 & $55.6(10)$ & 0.659 \\
\hline Obesity & $35.3(6)$ & 0.680 & $38.9(7)$ & 0.362 \\
\hline \multicolumn{5}{|c|}{ Penile dynamic duplex ultrasound improvement } \\
\hline & PSV Post-LiSWT \% (n) & $p$ value & EDV Post-LiSWT \% (n) & $p$ value \\
\hline HTA & $57.1(12)$ & 0.260 & $64.7(11)$ & 1.000 \\
\hline Diabetes & $23.8(5)$ & 0.548 & $23.5(4)$ & 0.640 \\
\hline Tobacco & $23.8(5)$ & 0.549 & $29.4(5)$ & 0.140 \\
\hline Dyslipidemia & $61.9(13)$ & 1.000 & $70.6(12)$ & 0.194 \\
\hline Obesity & $38.1(8)$ & 0.269 & $35.3(6)$ & 0.680 \\
\hline \multicolumn{5}{|c|}{ PDE5i treatment improvement } \\
\hline HTA & 40.0 & 2 & 0.312 & \\
\hline Diabetes & 0.0 & 0 & 0.274 & \\
\hline Tobacco & 20.0 & 1 & 1.000 & \\
\hline Dyslipidemia & 60.0 & 3 & 1.000 & \\
\hline Obesity & 20.0 & 1 & 0.642 & \\
\hline \multicolumn{5}{|c|}{ PDE5i response improvement } \\
\hline HTA & 44.4 & 4 & 0.200 & \\
\hline Diabetes & 0.0 & 0 & $* 0.027$ & \\
\hline Tobacco & 22.2 & 2 & 1.000 & \\
\hline Dyslipidemia & 55.6 & 5 & 1.000 & \\
\hline Obesity & 22.2 & 2 & 0.661 & \\
\hline \multicolumn{5}{|c|}{$\begin{array}{l}\text { ED: Erectile dysfunction; IIEF-5: international index of erectile function ( } 5 \text { questions); LiSWT: Low-intensity shock wave therapy; } \\
\text { Post-RP: Post radical prostatectomy; PSV: Peak systolic velocity; EDV: End-diastolic velocity; PDE5i: Phosphodiesterase type } 5 \\
\text { inhibitors. } \\
\text { * Statistical significance with } p<0.05\end{array}$} \\
\hline
\end{tabular}

treatment, mean IIEF-ED (erectile function domain) significantly improved from $13.5 \pm 4.1$ to $20.9 \pm$ $5.8(\mathrm{p}<0.001)(2)$. Later, this group conducted a randomized, double-blind, sham controlled study with 67 men. At one-month post-treatment, the mean IIEF-ED increased by 6.7 points in the treated group while in the sham group increased by 3.0 points ( $p 0.0322$ ) (4). Another randomized, doubleblind, placebo-controlled study by Yee et al, with 58 men, concluded that LiSWT presented significant improvement at 4 weeks post-treatment only in patients with severe ED (IIEF-ED improvement in LiSWT group was $10.1 \pm 4.1$, in placebo group was $3.2 \pm 3.3$ (p 0.003)) (5). Ruffo et al reported a study with 31 patients with mild to moderate ED.that achieved significant improvement in IIEF-ED: baseline mean IIEF-ED was 16.54 \pm 6.35 , at one-month post-treatment was $21.13 \pm 6.31($ p 0.0075$)$ and, at three-month was $21.03 \pm$ 6.38 (p 0.0096) (6). A meta-analysis conducted by Lu et al., comprising 14 studies including 833 patients revealed that LiSWT could significantly improve IIEF (mean difference 2.00; p < 0.0001) (7). Another meta-analysis conducted by Clavijo et al., comprising seven randomized controlled trials involving 602 patients, also reported a statistically significant improvement in pooled change in IIEF-ED score from baseline to follow-up in men treated with LiSWT compared with those receiving sham therapy (6.40 points; $95 \% \mathrm{CI}$ 1.78-11.02; p < 0.001 vs 1.65 points; 95 CI 0.92-2.39; p < 0.0001 ; between-group difference p 0.047) (8). Our results are in line with previous reported studies. Overall baseline median IIEF-5 was 14, at six weeks post-treatment was 16 ( $\mathrm{p}<0.001)$ and, at 3 months 18 ( $p<0.001)$, corresponding to an improvement of $68 \%$ and $72 \%$ respectively. At 3 months, median IIEF-5 actually changed category from mild-to-moderate to mild. Lu et al. (7) in his metaanalysis also reported a good therapeutic effect by 3 months, suggesting that changes induced by LiSWT may not be immediate but 
Table 5.

Results comparison.

\begin{tabular}{|c|c|c|c|c|}
\hline \multicolumn{5}{|l|}{ IIEF-5 improvement } \\
\hline 6 weeks & 6 weeks & 3 months & 3 months & $p$ value \\
\hline Post-LiSWT \% (n) & Post-LiSWT \% (n) & Post-LiSWT \% (n) & Post-LiSWT \% (n) & \\
\hline ED > 24 months & ED $\leq 24$ months & ED > 24 months & ED > 24 months & \\
\hline $69.2(9)$ & $66.7(8)$ & $61.5(8)$ & $83.3(10)$ & 0.378 \\
\hline \multicolumn{5}{|c|}{ Penile dynamic duplex ultrasound improvement } \\
\hline PSV & PSV & $p$ value & EDV & $p$ value \\
\hline Post-LiSWT \% (n) & Post-LiSWT \% (n) & Post-LiSWT \% (n) & Post-LiSWT \% (n) & \\
\hline ED > 24 months & ED $\leq 24$ months & ED > 24 months & ED > 24 months & \\
\hline $84.6(11)$ & $83.3(10)$ & $61.5(8)$ & $75.0(9)$ & 0.673 \\
\hline \multicolumn{5}{|c|}{ PDE5i treatment improvement } \\
\hline & $\mathrm{ED} \leq 24$ months & $\mathrm{ED}>24$ months & & \\
\hline & $7.7(1)$ & $33.3(4)$ & 0.160 & \\
\hline \multicolumn{5}{|c|}{ PDE5i response improvement } \\
\hline & Post-LiSWT \% (n) & Post-LiSWT \% (n) & $p$ value & \\
\hline & $\mathrm{ED} \leq 24$ months & ED $>24$ months & & \\
\hline & $15.4(2)$ & $58.3(7)$ & $* 0.041$ & \\
\hline \multicolumn{5}{|c|}{$\begin{array}{l}\text { IIEF-5: international index of erectile function (5 questions); LiSWT: Low-intensity shock wave therapy; IQR: Interquartile range; } \\
\text { SD: Standard deviation; PSV: Peak systolic velocity; EDV: End-diastolic velocity; PDE5i: Phosphodiesterase type } 5 \text { inhibitors. } \\
\text { * Statistical significance with } p<0.05\end{array}$} \\
\hline
\end{tabular}

ence between the two groups (PSV improvement in group 1 was $84.6 \%$ vs $83.3 \%$ in group 2; p 1.000 and EDV improvement in group 1 was $61.5 \%$ vs $75.0 \%$ in group $2 ; \mathrm{p} 0.673)$. The majority of published studies addressed treatment outcomes with sexual function and quality of live questionnaires but not many have assessed penile hemodynamics in patients treated with LiSWT for ED. Kalyvianakis et al., in a doubleblinded, randomized, sham controlled trial with 46 patients, like in our study, used PDDU to evaluate patients at 3 months post-treatment and reported a mean change in PSV of $4.5 \mathrm{~cm} / \mathrm{s}$ and $0.6 \mathrm{~cm} / \mathrm{s}$ for the treatment and sham-control groups, respectively $(\mathrm{p}<0.001)$ (12). Other studies, namely Vardi et al (4) and Kitrey et al. (13) also assessed penile hemodynamics with another technique using the

Table 6.

Patient questionnaire.

\begin{tabular}{|lccc|}
\hline & Satisfaction & Recommendation & Adverse effects \\
\hline & $\%(\mathbf{n})$ & $\%(\mathbf{n})$ & $\%(\mathbf{n})$ \\
\hline Total & $76.0(19)$ & $80.0(20)$ & $0.0(0)$ \\
\hline $\mathrm{ED} \leq 24$ months & $76.9(10)$ & $84.6(11)$ & $0.0(0)$ \\
\hline $\mathrm{ED}>24$ months & $75.0(9)$ & $75.0(9)$ & $0.0(0)$ \\
\hline
\end{tabular}

rather delayed in time. Our subgroups analysis by length of disease duration showed no significant difference improvement in IIEF- 5 between groups at 6 weeks or at 3 months follow-up post-treatment (p 1.00 and p 0.378 respectively), suggesting that time of ED do not alter treatment outcomes. Pelayo-Nieto et al, in a study with 15 patients reported an overall improvement in IIEF-ED of $80 \%(14.23$ vs $19.69 ; \mathrm{p}<0.0013)$ and no influence of ED duration was found using a cut-off of 3 years $(p<$ 0.20) (9). In a multicenter open-label prospective study with 58 patients, Reisman et al. reported an overall improvement of $81.03 \%$ in IIEF-ED (IIEF-ED average increase $7.5 \pm 4.7 ; p<0.001$ ). Furthermore, a only moderate negative Pearson correlation coefficient of -0.62 was found between the duration of ED and success of treatment, showing satisfactory success rates in cases of ED up to 10 years of duration (10). Also, Bechara et al, in a study with 50 patients, concluded that time of ED did not influenced the results (11). Our study evaluation relied not only in subjective patient questionnaire like IIEF-5, but also assessed penile hemodynamics with a tangible tool like penile Doppler ultrasound. Our overall results showed a significant improvement both in mean PSV $(29.3 \mathrm{~cm} / \mathrm{s}$ vs $35.9 \mathrm{~cm} / \mathrm{s} ; \mathrm{p}$ 0.001) and mean EDV $(2.6 \mathrm{~cm} / \mathrm{s}$ vs $1.3 \mathrm{~cm} / \mathrm{s} ; \mathrm{p} 0.015)$ with both post-treatment values within normal ranges. Also, subgroups analysis by length of disease duration showed no significant differ- flow mediated dilation. Both groups reported significant improvement $(p<0.0001)$. These results show that LiSWT indeed produces changes in penile vascularization associated with improved hemodynamics. Another endpoint of our study was to evaluate the influence of LiSWT in PDE5i response and if patients were able to leave this medication after treatment. Significant results were seen in patients who still needed PDE5i after treatment, because their response to medication improved (overall improvement of $36.0 \%$; p 0.004). Significant difference was present between groups ( $p$ 0.041), being group 2 the major responsible for this improvement, showing that patients with longer ED responded better to PDE5i after LiSWT. It is a fact that these patients were all on PDE5i previously, thus, more used to it and more aware, and this might have influenced the results. At the end of the study 5 patients (20\%) were able to leave permanently PDEi5 and achieve spontaneous erections, nevertheless this was not statistically significative ( $p$ 0.063). Others have evaluated the effect of LiSWT on PDE5i response. Grueenwald et al., in a study with 29 men with severe ED and poor response to PDEi5, showed that one month post-treatment, 34\% of patients returned to sexual activity without the necessity for pharmacotherapy (14).

Our protocol included having tadalafil $5 \mathrm{mg}$ daily. Although it could induce a bias in the results, following the concept of angiogenesis and neovascularization associated with LiSWT, concomitant PDE5i produces a continuous local stimulus that might contribute to a synergic effect with LiSWT and potentiate global response in the best interest for the patient. Kitrey et al., in a prospective randomized, double-blind sham-controlled study with 55 patients, also used PDEi5 during LiSWT and showed that $54 \%$ of these patients achieved erections hard enough for penetration, in comparison of $0 \%$ of the sham group (PDE5i only) (13). The meta-analysis per- 
formed by Lu et al. showed that the IIEF increased more significantly in the group with LiSWT combined with PDE5i use (mean difference: 4.20; 95\% CI, 0.16-8.24; p 0.04 ), supporting the use of combination therapy (7).

When stratified by type of ED, our results showed that patients with vasculogenic ED, whether arteriogenic and/or venous leak, presented the best results, regarding IIEF- 5 at 6 weeks post-treatment and, patients with ED post radical prostatectomy, the worse results ( $\mathrm{p} 0.021$ ). There is a consensus in the literature that the major suggested mechanism of action of LiSWT is by angiogenesis and neovascularization, and that explains why patients with vasculogenic ED are expected to be the best candidates to this treatment. Nevertheless, assuming mechanisms of action like nerve regeneration, other patients may be candidates. Frey et al., in a study with 16 patients who underwent nerve-sparring robotic radical prostatectomy, reported significant changes in IIEF-5 post-LiSWT, with a median increase of +3.5 at one-month ( $p$ 0.0049) and, +1 at one-year post-treatment ( $p$ 0.046), nevertheless, the majority of patients achieved only marginal improvements in ED category (15). ED postprostatectomy is usually a severe and complex side-effect, caused by direct trauma, stretching, heating, ischemia and local inflammation of the cavernous nerves (16). Nerve damage results in impaired erections and inadequate penile oxygenation, leading to smooth muscle apoptosis and fibrosis (17). In this setting, it may be too ambitious expecting evident results with LiSWT, but it may have a role as adjunctive therapy in penile rehabilitation.

When looking for the influence of ED risk factors on LiSWT outcomes, our study showed that diabetic patients presented the worse results. In fact, statistical significance was found in PDEi5 response, where diabetic patients didn't show any improvement ( $\mathrm{p}$ 0.027). Reisman et al., comparing diabetic and non-diabetic patients, had a success rate $25 \%$ higher in the latter group ( $70.83 \%$ vs $88.24 \%$ respectively) (10). Hisasue et al, in subgroup analysis by comorbidities, found worse results in diabetic patients with only $3 / 10$ achieving a score of 3 in Erection Hardness Score (18). These results all together suggest a negative impact of diabetes on the efficacy of LiSWT.

Contemporary LiSWT machines can be divided into 3 main types based on the mechanism of shock waves namely electrohydraulic, electromagnetic and piezoelectric. The majority of studies have used the first two types. The piezoelectric device differs from the others in that it offers full organ coverage and higher treatment parameters. Motil et al., like in our study, used a piezoelectric machine and reported an average IIEF-5 score improvement from 14.4 baseline to18.6 at 1-month post-treatment. A total of 75 patients were treated and they had PDE5i during treatment (19). Fojecki et al., in a randomized, double-blinded, sham-controlled study with 126 patients, also used a piezoelectric device, and reported success rates based on the IIEF-EF score of 38.3\% in the sham group and $37.9 \%$ in the active group $(\mathrm{OR}=95$, $95 \% \mathrm{CI}=0.45-2.02$, p 0.902), showing no clinical relevant effect of LiSWT (20). Although using the same device, Fojecki et al., delivered less energy to the penis, using an EFD of $0.09 \mathrm{~mJ} / \mathrm{mm}^{2}$, in contrast Motil et al., like in our study an EFD of $0.160 \mathrm{~mJ} / \mathrm{mm}^{2}$ was used. Also, in the Fojecki study, patients had a 4-weeks wash-out period of PDEi5 and medication was not allowed during treatment. This protocol differences may be responsible for different outcomes between these studies, reinforcing the benefit of using adjuvant PDEi5 with LiSWT.

Limitations of our study are the absence of a sham-control arm, a small number of patients and the short follow-up period. Also, the concomitant use of PDE5i could induce a bias. Nevertheless, the strengths include being prospective, having evaluated penile hemodynamics in all patients with a tangible and reliable tool as PDDU, no limitation in inclusion criteria regarding type of ED and looking for cofounding factors that could influence treatment outcomes, specially duration of ED.

LiSWT is a safe, harmless, repeatable treatment modality for ED with good functional outcomes reported. Our results suggest that length of disease duration doesn't negatively influences treatment results. Also, concomitant use of PDE5i should be considered.

\section{References}

1. Feldman HA, Goldstein I, Hatzichristou DG, et al. Impotence and its medical and psychosocial correlates: results of the Massachusetts Male Aging Study. J Urol. 1994; 151:154.

2. Vardi Y, Appel B, Jacob G, et al. Can low intensity extracorporeal shockwave therapy improve erectile function? A 6-month followup pilot study in patients with organic erectile dysfunction. Eur Urol. 2010; 58:243-248.

3. Wang HJ, Cheng JH, Chuang YC. Potential applications of lowenergy shock waves in functional urology. Int J Urol. 2017; 24:573581.

4. Vardi Y, Appel B, Kilchevsky A, Gruenwald I. Does low intensity extracorporeal shockwave therapy have a physiological effect on erectile function? Short-term results of a randomized, double-blind, sham controlled study. J Urol. 2012; 187:1769-1775.

5. Yee CH, Chan ES, Hou SS, Ng CF. Extracorporeal shockwave therapy in the treatment of erectile dysfunction: a prospective, randomized, double-blinded, placebo controlled study. Int J Urol. 2014; 21:1041-1045.

6. Ruffo A, Capece M, Prezioso D, et al. Safety and efficacy of low intensity shockwave (LISW) treatment in patients with erectile dysfunction. Int Braz J Urol. 2015; 41:967-974.

7. Lu Z, Lin G, Reed-Maldonado A, et al. Low-intensity extracorporeal shock wave treatment improves erectile function: a systematic review and meta-analysis. Eur Urol. 2017; 71:223-233.

8. Clavijo RI, Kohn TP, Kohn JR, Ramasamy R. Effects of low-intensity extra-corporeal shockwave therapy on erectile dysfunction: a systematic review and meta-Analysis. J Sex Med. 2017; 14:27-35.

9. Pelayo-Nieto M, Linden-Castro E, Alias-Melgar A, et al. Linear shock wave therapy in the treatment of erectile dysfunction. Actas Urol Esp. 2015; 39:456-459.

10. Reisman Y, Hind A, Varaneckas A, Motil I. Initial experience with linear focused shockwave treatment for erectile dysfunction: a 6-month follow-up pilot study. Int J Impot Res. 2015; 27:108-112.

11. Bechara A, Casabé A, De Bonis W, Gomez Ciciclia P. Twelvemonth efficacy and safety of low-intensity shockwave therapy for erectile dysfunction in patients who do not respond to phosphodiesterase type 5 inhibitors. Sex Med. 2016; 4:e225-e232. 
12. Kalyvianakis D, Hatzichristou D. Low-intensity shockwave therapy improves hemodynamic parameters in patients with vasculogenic erectile dysfunction: a triplex ultrasonography-based shamcontrolled trial. J Sex Med. 2017; 14:891-897.

13. Kitrey ND, Gruenwald I, Appel B, et al. Penile low intensity shock wave treatment is able to shift PDE5i nonresponders to responders: a double-blind, sham controlled study. J Urol. 2016; 195:1550-1555.

14. Gruenwald I, Appel B, Vardi Y. Low-intensity extracorporeal shock wave therapy-a novel effective treatment for erectile dysfunction in severe ED patients who respond poorly to PDE5 inhibitor therapy. J Sex Med. 2012; 9:259-264.

15. Frey A, Sonksen J, Fode M. Low-intensity extracorporeal shockwave therapy in the treatment of postprostatectomy erectile dysfunction: a pilot study. Scand J Urol 2016; 50:123-127.

16. Burnett AL. Rationale for cavernous nerve restorative therapy to preserve erectile function after radical prostatectomy. Urology 2003; 6:491-497.

17. Iacono F, Gianella R, Somma P, et al. Histological alterations in cavernous tissue after radical prostatectomy. J Urol. 2005; 173:1673-1676.

18. Hisasue S, China T, Horiuchi A, et al. Impact of aging and comorbidity on the efficacy of low-intensity shock wave therapy for erectile dysfunction. Int J Urol. 2016; 23:80-84

19. Motil I, Kubis I, Sramkova, T. Treatment of vasculogenic erectile dysfunction with Piezowave 2 device. Application of low intensity shockwaves using novel linear shockwave tissue coverage (LSTC$\left.E D^{\circledR}\right)$ technique. A prospective, multicentric, placebo-controlled study. Adv Sex Med. 2016; 6:15-18.

20. Fojecki GL, Tiessen S, Osther PJS. Effect of low-energy linear shockwave therapy on erectile dysfunction - a double-blinded, shamcontrolled, randomized clinical trial. J Sex Med 2017; 14:106-1.

\section{Correspondence}

Pedro Simoes de Oliveira, MD (Corresponding Author) pedrosimoesdeoliveira@gmail.com

Tiago Ribeiro de Oliveira,MD tiagoribeirooliveira@sapo.pt

Álvaro Nunes, MD

alvaronunes@portugalmail.pt

Francisco Martins, MD

faemartins@gmail.com

Tomé Lopes, MD

tomematoslopes@gmail.com

SCI-Centro de Urologia

Av. Defensores de Chaves, 83, 1º, 1000-115, Lisbon, Portugal 